\title{
Design of mm-wave slow-wave devices with sheet and hollow electron beams
}

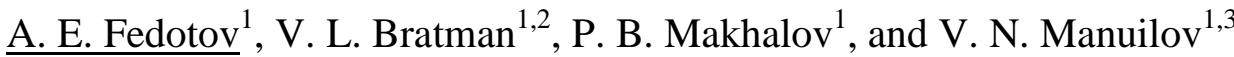 \\ ${ }^{1}$ Institute of Applied Physics, Nizhny Novgorod, Russia, fedotov@appl.sci-nnov.ru \\ ${ }^{2}$ Ariel University, Ariel, Israel \\ ${ }^{3}$ Nizhny Novgorod State University, Nizhny Novgorod, Russia
}

\begin{abstract}
The frequency increase of the slow-wave electron devices is accompanied by inevitable increase of the electron current density and ohmic losses which strongly restricts the attainable power. In recent years, the use of the spatially-developed sheet electron beams is considered as the major way to develop the medium-power slow-wave devices at $\mathrm{mm}$ and sub$\mathrm{THz}$ waves. Hollow electron beams is another configuration which could be used for this purpose. The designs of the oscillators and amplifiers in Ka-band and W-band with both sheet and hollow electron beams are considered and compared.
\end{abstract}

\section{Introduction}

The development of the powerful amplifiers in the millimeter and sub-terahertz wavelength ranges is one of the most topical problem in vacuum microwave electronics. In particular, millimeter-wave amplifiers with an output power of several hundred watts are of interest for spectroscopy, telecommunications, remote sensing, and also as preamplifiers for even more powerful gyro-amplifiers. The difficulty of development of traveling wave tubes (TWT) and extended-interaction klystrons (EIK) with the required parameters is caused largely by a very high required beam current density (up to $1 \mathrm{kA} / \mathrm{cm}^{2}$ ) if conventional electron beam with pencil-like configuration is used. Due to evanescent nature of the synchronous space harmonic of the operating mode, this beam with high energy density should be guided in close proximity to the surface of the slow-wave structure in order to provide efficient electron-wave interaction. The non-perfection of the beam guiding system and high microwave fields inevitably results in some current deposition and, hence, a high heat load on the small elements of the slow-wave structure, in addition to ohmic losses. In order to alleviate the thermal regime of the devices, a use of planar sheet electron beams for millimeter-wave TWTs and EIKs are extensively studied in recent years [1-3]. The large width of the planar beam allows decrease of the required current density but increase of the total electron current. Therefore, the CW or average output power for the planar-beam slow-wave amplifier can be significantly higher than for the pencil-beam device. An alternative configuration of the spatially-developed electron beam is a cylindrical hollow thin-wall beam [4,5]. In this paper we consider both these options for development of the slow-wave device with medium power at the millimeter wavelengths.

\section{Design of sheet-beam slow-wave TWT and BWO}

Two key points in the development of the sheetbeam slow-wave devices are slow-wave structure and electron optical system. Presented design is based on a slow-wave structure consisting of two gratings shifted by half period relative to each other (Fig. 1). This structure is widely explored in recent years since it is simple, wideband, easy to manufacture and compatible with a planar electron beam [6]. The sheet beam can be provided by quasi-Pierce electron gun and then solenoidally focused and guided. The beam guiding in the solenoidal magnetic field seems to be simpler than periodic-permanent-magnet focusing system which is traditional for pencil-beam devices at longer waves. To reduce the magnetic compression ratio, a thermionic cathode with high current density of $30 \mathrm{~A} / \mathrm{cm}^{2}$ can be used, similar to cathodes of lowpower submillimeter backward-wave oscillators, clinotrons and orotrons. Simulations predict that the beam which is $0.2 \mathrm{~mm}$ thick and $1.8 \mathrm{~mm}$ wide with a current of $300 \mathrm{~mA}$ (current density $80 \mathrm{~A} / \mathrm{cm}^{2}$ ) can compressed and guided between gratings in $0.65 \mathrm{~T}$ magnetic field with less than $5 \%$ of the total current being lost. Simulation made for W-beam TWT shows the possibility of obtaining an output power of $250 \mathrm{~W}$ at a voltage of $20 \mathrm{kV}$. The calculated gain is $26 \mathrm{~dB}$ in the linear regime and about of $21 \mathrm{~dB}$ in the saturation. For initial experimental verification of the electronoptical system, a BWO with similar periodic structure was designed with the power of $200-300 \mathrm{~W}$ and $7 \%$ tuning band (Fig. 2). The same cathode makes it possible to design the Ka-band BWO and TWT with 500$\mathrm{W}$ output power using beam guiding in the uniform magnetic field, without beam compression.

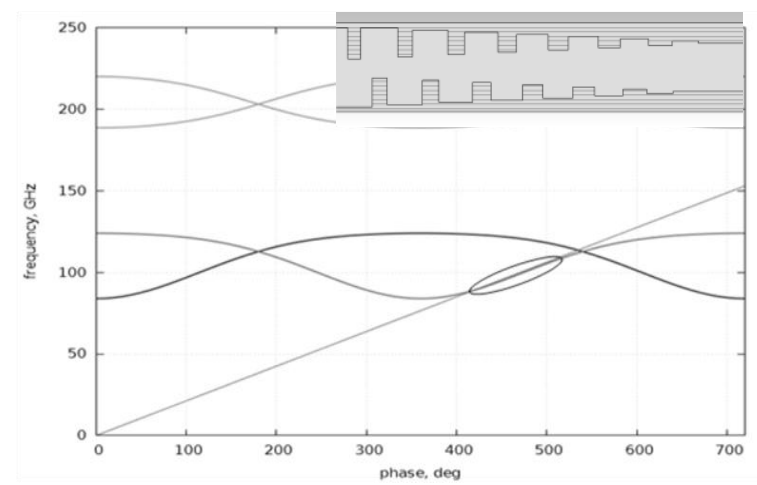

Fig. 1. Calculated dispersion of the operating mode for designed W-band TWT and line of synchronism with $20-\mathrm{kV}$ electron beam. Inset: the staggered-gratings slow-wave structure with the smooth taper of the gratings near the collector end of the interaction region. 


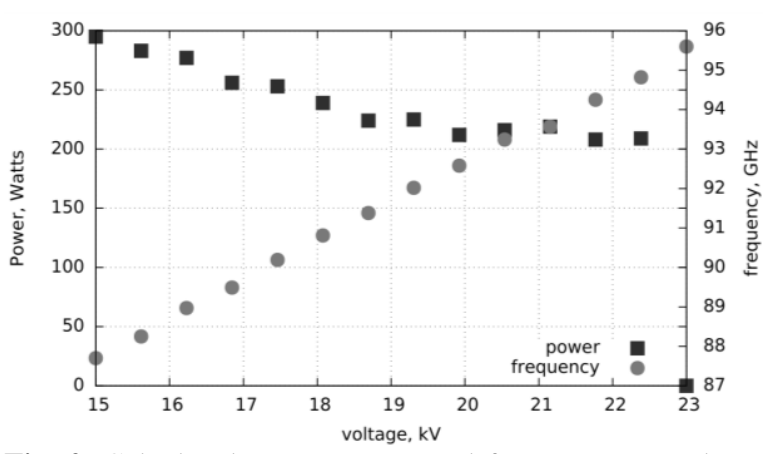

Fig. 2. Calculated output power and frequency vs. voltage for sheet-beam W-band BWO with the staggered-gratings slow-wave structure.

\section{Design of hollow-beam BWOs}

The use of hollow electron beams permits a significant increase in the diameter of the beam tunnel in comparison with the conventional pencil-beam slowwave electron devices. Hollow beam can efficiently interact with a lowest azimuthally-symmetric TM mode of the azimuthally-symmetric slow-wave structure. This configuration is similar to the relativistic high-current electron devices. However, the slowwave structures with deep corrugation should be used in non-relativistic devices due to weak electron-wave coupling in the millimeter wavelength range. W-band and Q-band axisymmetric BWOs has been designed on the base of $30 \mathrm{kV} / 1 \mathrm{~A}$ hollow thin-wall electron beam with an outer diameters of $1.6 \mathrm{~mm}$ and $3.4 \mathrm{~mm}$, respectively. This beam is produced in a Pierce-like electron gun with the hundredfold magnetic compression (Fig. 3). Simulations based on the averaged equations and 3D PIC-code predict an output power of 300-500 $\mathrm{W}$ (depending on the beam wall thickness) for W-band BWO and about of $800 \mathrm{~W}$ for Q-band BWO. $5 \%$ frequency tuning is simulated for the Wband device. To reduce ohmic losses, the wave reflections at the collector end of slow-wave structures are minimized by using specially designed last matching tooth (Fig. 4).

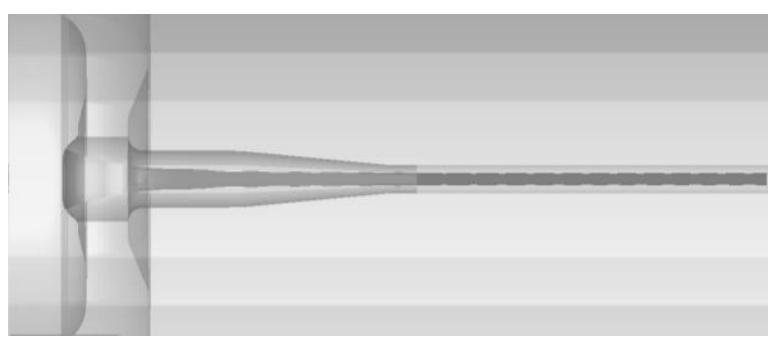

Fig. 3. Designed electron gun producing $30-\mathrm{kV}, 1$ A hollow electron beam with simulated electron trajectories.

In conclusion, let us compare both configurations of the spatially-developed electron beams. The slowwave structures for sheet beam devices are easier for manufacturing, and they could provide wider frequency band. The electron-wave coupling for sheet-beam devices could be stronger since electrons travel between two gratings, while for hollow beam there is single grating at the outer side of the beam. On the other hand, the formation of the sheet electron beam is difficult due to diocotron instability, but this difficulty can be avoided if cathodes with high emission density are used.

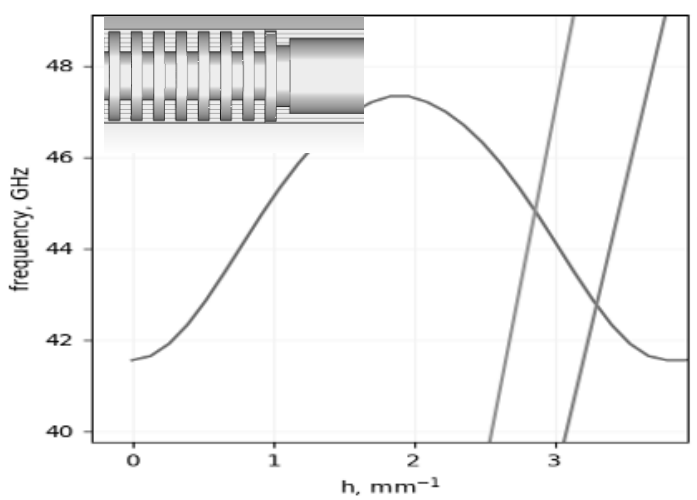

Fig. 4. Calculated dispersion of the operating mode for designed Q-band BWO and line of synchronism with 20- and $30-\mathrm{kV}$ hollow beams. Inset: the axisymmetric slow-wave structure with the matching tooth at the collector end.

The development of the planar-beam devices is supported by Russian Science Foundation under grant No. 18-79-10252. The development of the hollowbeam devices is supported by RFBR under grant No. 16-08-01174.

\section{References}

1. Pershing, D. E., Nguyen, K. T., Abe, D. K., Wright, E., Larsen, P. B., Pasour, J., Cooke, S. J., Balkcum, A., Wood, F. N., Myers, R. E., and Levush, B. Demonstration of a wideband 10-kW Ka-band sheet beam TWT amplifier. // IEEE Transactions on Electron Devices. 2014. V. 61, No. 6. P. 1637-1642.

2. Pasour, J., Wright, E., Nguyen, K. T., Balkcum, A., Wood, F. N., Myers, R. E., and Levush, B. Demonstration of a multikilowatt, solenoidally focused sheet beam amplifier at $94 \mathrm{GHz}$. // IEEE Transactions on Electron Devices. 2014. V. 61, No. 6. P. 1630-1636.

3. Karetnikova, T. A., Rozhnev, A. G., Ryskin, N. M., Fedotov, A. E., Mishakin, S. V., and Ginzburg, N. S. Gain analysis of a $0.2-\mathrm{THz}$ traveling-wave tube with sheet electron beam and staggered grating slow wave structure. // IEEE Transactions on Electron Devices. 2018. V. 65, No. 6. P. 2129-2134.

4. Bratman, V. L., Fedotov, A.E., and Makhalov, P. B. High-frequency devices with weakly-relativistic hollow thin-wall electron beams. // Physics of Plasmas. 2012. V. 19, No. 2. Art. 020704.

5. Bratman, V. L., Fedotov, A. E., Makhalov, P. B., and Manuilov, V. N. Design and numerical analysis of Wband oscillators with hollow electron beam. // IEEE Transactions on Electron Devices. 2014. Vol. 61, No. 6. P. 17951799.

6. Shin, Y. M., Barnett, L. R., and Luhmann, N. C. Phase-shifted traveling-wave-tube circuit for ultrawideband high-power submillimeter-wave generation. // IEEE Transactions on Electron Devices. 2009. V. 56, No. 5. P. 706712 . 